\title{
Radioactivity backgrounds in ZEPLIN-III
}

\author{
H.M. Araújo ${ }^{a, *}$, D.Yu. Akimov ${ }^{\mathrm{b}}$, E.J. Barnes ${ }^{\mathrm{c}}$, V.A. Belov ${ }^{\mathrm{b}}$, A. Bewick ${ }^{\mathrm{a}}$, A.A. Burenkov ${ }^{\mathrm{b}}$, V. Chepel $^{\mathrm{d}}$, \\ A. Currie ${ }^{\mathrm{a}}$, L. DeViveiros ${ }^{\mathrm{d}}$, B. Edwards ${ }^{\mathrm{e}}$, C. Ghag ${ }^{\mathrm{c}}$, A. Hollingsworth ${ }^{\mathrm{c}}$, M. Horn ${ }^{\mathrm{a}}$, G.E. Kalmus ${ }^{\mathrm{e}}$, \\ A.S. Kobyakin ${ }^{\mathrm{b}}$, A.G. Kovalenko ${ }^{\mathrm{b}}$, V.N. Lebedenko ${ }^{\mathrm{a}}$, A. Lindote ${ }^{\mathrm{d}, \mathrm{e}}$, M.I. Lopes ${ }^{\mathrm{d}}$, R. Lüscher ${ }^{\mathrm{e}}$, \\ P. Majewski ${ }^{\mathrm{e}}$, A.St J. Murphy' ${ }^{\mathrm{c}}$, F. Neves ${ }^{\mathrm{d}, \mathrm{a}}$, S.M. Paling ${ }^{\mathrm{e}}$, J. Pinto da Cunha $^{\mathrm{d}}$, R. Preece ${ }^{\mathrm{e}}$, J.J. Quenby ${ }^{\mathrm{a}}$, \\ L. Reichhart ${ }^{\mathrm{c}}$, P.R. Scovell ${ }^{\mathrm{c}}$, C. Silva ${ }^{\mathrm{d}}$, V.N. Solovov ${ }^{\mathrm{d}}$, N.J.T. Smith ${ }^{\mathrm{e}}$, P.F. Smith ${ }^{\mathrm{e}}$, V.N. Stekhanov ${ }^{\mathrm{b}}$, \\ T.J. Sumner ${ }^{\mathrm{a}}$, C. Thorne ${ }^{\mathrm{a}}$, R.J. Walker ${ }^{\mathrm{a}}$ \\ ${ }^{a}$ High Energy Physics group, Blackett Laboratory, Imperial College London, UK \\ ${ }^{b}$ Institute for Theoretical and Experimental Physics, Moscow, Russia \\ ${ }^{c}$ School of Physics \& Astronomy, SUPA University of Edinburgh, UK \\ ${ }^{d}$ LIP-Coimbra \& 3 Department of Physics of the University of Coimbra, Portugal \\ ${ }^{e}$ Particle Physics Department, STFC Rutherford Appleton Laboratory, Chilton, UK
}

\begin{abstract}
We examine electron and nuclear recoil backgrounds from radioactivity in the ZEPLIN-III dark matter experiment at Boulby. The rate of low-energy electron recoils in the liquid xenon WIMP target is $0.75 \pm 0.05$ events $/ \mathrm{kg} / \mathrm{day} / \mathrm{keV}$, which represents a 20 -fold improvement over the rate observed during the first science run. Energy and spatial distributions agree with those predicted by component-level Monte Carlo simulations propagating the effects of the radiological contamination measured for materials employed in the experiment. Neutron elastic scattering is predicted to yield $3.05 \pm 0.5$ nuclear recoils with energy $5-50 \mathrm{keV}$ per year, which translates to an expectation of 0.4 events in a 1-year dataset in anti-coincidence with the veto detector for realistic signal acceptance. Less obvious background sources are discussed, especially in the context of future experiments. These include contamination of scintillation pulses with Cherenkov light from Compton electrons and from $\beta$ activity internal to photomultipliers, which can increase the size and lower the apparent time constant of the scintillation response. Another challenge is posed by multiple-scatter $\gamma$-rays with one or more vertices in regions that yield no ionisation. If the discrimination power achieved in the first run can be replicated, ZEPLIN-III should reach a sensitivity of $\sim 1 \times 10^{-8} \mathrm{pb} \cdot$ year to the scalar WIMP-nucleon elastic cross-section, as originally conceived.
\end{abstract}

Keywords: Liquid xenon detectors, dark matter searches, ZEPLIN-III, radioactivity, background studies

\section{Introduction}

The ZEPLIN-III direct dark matter search has been operating at the Boulby underground laboratory (UK) under a rock overburden of $2,800 \mathrm{~m}$ water equivalent. The WIMP target is a xenon emission detector [1] which discriminates between electron and nuclear recoils by measuring the relative amount of prompt scintillation light and ionisation charge extracted from particle interactions in the liquid xenon (LXe) phase; the ionisation is trans-

\footnotetext{
* Corresponding author

Email address: h.araujo@imperial.ac.uk (H.M. Araújo)
}

Preprint submitted to Astroparticle Physics duced into a second optical signal via electroluminescence in the thin vapour layer above the liquid. Both the primary (S1) and secondary (S2) scintillation pulses are detected by an array of 31 photomultipliers immersed in the liquid. Key components of the experiment are shown in Figure 1, for details on the design and construction of the system we refer the reader to Refs. 2, 3, 3, 4, 5].

After deployment underground in early 2007, an 83-day run produced competitive limits for WIMPnucleon scattering cross-sections [6, 7, 8]. In this first phase the background of the experiment was dominated by the photomultiplier array by a significant factor - both in electron recoils from $\gamma$-rays and in nuclear recoils from neutrons. A develop-

September 17, 2018 


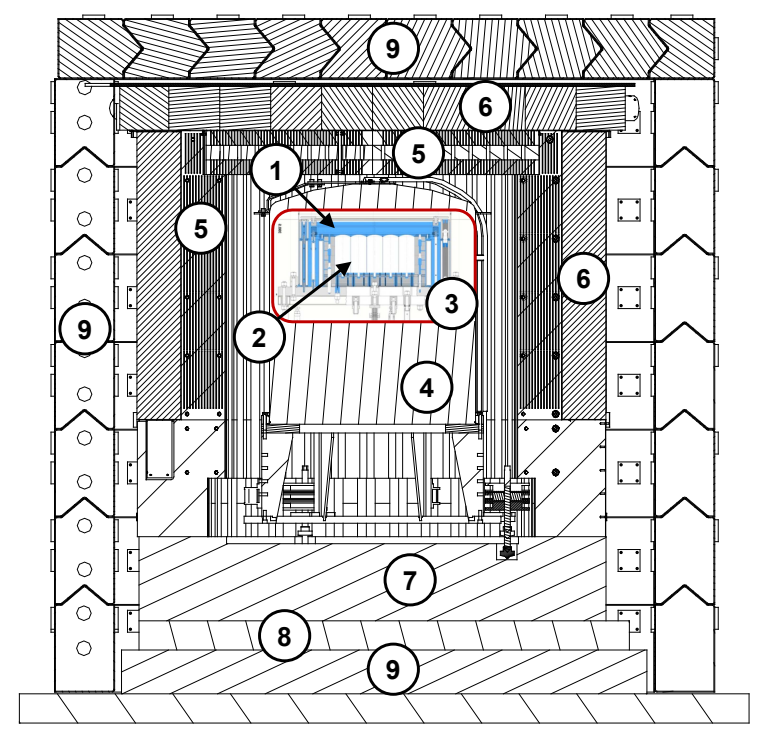

Figure 1: Schematic diagram of the ZEPLIN-III experiment. 1: WIMP target (LXe shown in blue); 2: photomultiplier array; 3: xenon chamber; 4: vacuum cryostat; 5: Gdloaded polypropylene; 6: veto scintillator modules; 7-bare polypropylene; 8-copper flooring; 9-lead shielding.

ment programme was thus undertaken with industry in order to develop new phototubes targeting an order of magnitude reduction in radioactivity. After upgrade of the photomultipliers and the addition of a veto system around the main target [4, 5], the experiment returned to taking WIMP search data in mid 2010. The first aim of this article is to describe the radioactivity backgrounds which determine the sensitivity of the rare event search ahead of forthcoming results from the second run.

We highlight also that many background studies focus solely on simple event topologies quantifiable by Monte Carlo simulation, but often overlook other important factors. Although the requirement of negligible neutron background drives the design of most dark matter experiments, in fact this threat materialises only rarely. In two-phase xenon detectors, multiple scattering $\gamma$-rays can be challenging when one of the vertices occurs in a region which yields no ionisation; in this instance the prompt scintillation signals for the multiple vertices are time-coincident and difficult to distinguish, and the charge-to-light ratio (S2/S1) is low, more typical of nuclear recoils. In another example, when estimating photomultiplier backgrounds, many studies consider only high-energy $\gamma$-rays as contribut- ing significantly to electron recoil background; we present evidence here that $\beta$-induced signals generated internally (involving, for example, Cherenkov emission from the phototube envelopes) should also be assessed carefully.

Besides their sensitivity in the scintillation and ionisation channels (see, e.g., Ref. [9] for an emphatic demonstration of the latter), two-phase xenon detectors hold great promise for next generation dark matter searches due to the ability to self-shield against external backgrounds. The accurate reconstruction of the position of interactions in three dimensions allows a sacrificial LXe volume to be defined which shields an inner 'fiducial' mass from most sources of radioactivity. ZEPLINII was the first WIMP experiment to operate using this technology 10], soon followed by XENON10 at Gran Sasso 11] and ZEPLIN-III at Boulby [6]. XENON100 12] already benefits from self-shielding very significantly, and even more so will the upcoming LUX350 experiment at SUSEL [13]. Twophase xenon experiments have been at the forefront of WIMP sensitivity and systems with tonne-scale fiducial mass could probe most of the parameter space favoured by constrained minimal supersymmetry (cMSSM) and by other extensions to the standard model. With this work we wish to summarise a decade of experience in designing, building and operating ZEPLIN-III, and thus inform the design of next-generation WIMP experiments.

This article is organised as follows. Ordinary, single-vertex electron recoil backgrounds are treated in Section 2, where ZEPLIN-III data are confronted with an overall prediction built up from component-level simulations. In Section 3 we discuss event topologies that may degrade the discrimination power of these instruments, such as $\gamma$-ray multiple scatters and $\beta^{-}$background internal to the photomultipliers. Having validated U/Th contamination levels in key components, we present calculations of neutron background in Section 4. Finally, we summarise our findings in Section 5. with the design of future systems in mind.

\section{Single-vertex electron recoil backgrounds}

Most low-energy electron recoils in the LXe result from interactions of $\gamma$-rays and $\beta$-particles from natural radioactivity. We begin by calculating absolute spectra of deposited energy from dominant sources, and compare these with ZEPLIN-III data acquired at Boulby in fully-shielded configuration. 
These signals can be discriminated from nuclear recoils to a large degree; it is reasonable to expect that a 'leakage' (mis-identification probability) of order 1:10,000 may be achieved for single-site interactions such as those from ${ }^{85} \mathrm{Kr} \beta$-particles within the LXe (the value eventually achieved in the first run for all interactions was 1:7,800 [14]). However, more complicated topologies must be considered, and these may not be rejected so efficiently. We analyse two such types in Section 3 .

The $\gamma$-ray background is dominated by primordial $\mathrm{U}$, Th and ${ }^{40} \mathrm{~K}$. We assume natural uranium $\left(0.956{ }^{238} \mathrm{U}, 0.044{ }^{235} \mathrm{U}\right)$ and that all chains are in secular equilibrium, except where indicated otherwise. To determine contamination levels, a large number of materials were radio-assayed over the lifetime of the project. All HPGe $\gamma$-ray spectroscopy was conducted at Boulby with an ORTEC GEM detector with a $2 \mathrm{~kg}$ crystal, reaching down to $\sim 0.5 \mathrm{ppb}$ sensitivity for $\mathrm{U}$ and $\mathrm{Th}$. Energy-dependent counting efficiencies were calculated from Monte Carlo simulations for each test geometry. These measurements were complemented by commercial mass spectrometry (ICP-MS) for several materials. We have also benefited from the extensive UKDMC database 15.

The 20 most intense $\gamma$-rays in each chain and the $1,461 \mathrm{keV}$ line from ${ }^{40} \mathrm{~K}$ are propagated using the GEANT4 Monte Carlo toolkit [16] to interactions in the LXe target. For internal backgrounds the ZEPLIN-III simulation model was used [2]; this was complemented by importing a detailed CAD solid model for calculations involving the veto, shielding and the laboratory rock.

\subsection{ZEPLIN-III measurements}

A 24-day long dataset was analysed to study the spatial and energy distribution of electron recoil background. This followed a neutron calibration by only a few weeks, and so isomeric transition (IT) $\gamma$-rays from activation of the xenon are still visible (mainly from ${ }^{129 m} \mathrm{Xe}, \mathrm{T}_{1 / 2}=8.88$ days, and ${ }^{131 \mathrm{~m}} \mathrm{Xe}, \mathrm{T}_{1 / 2}=11.8$ days). The data were collected as part of the WIMP search dataset and, for this analysis, the nuclear recoil signal region remained blinded up to $40 \mathrm{keVee} 1$ However, this has little impact on the electron recoil population. Events with

\footnotetext{
${ }^{1}$ LXe energy deposits are expressed in electron-equivalent 'keVee' units when calibrated by ${ }^{57} \mathrm{Co} 122 \mathrm{keV} \gamma$-rays, or in 'keVnr' when referring to nuclear recoil energy.
}
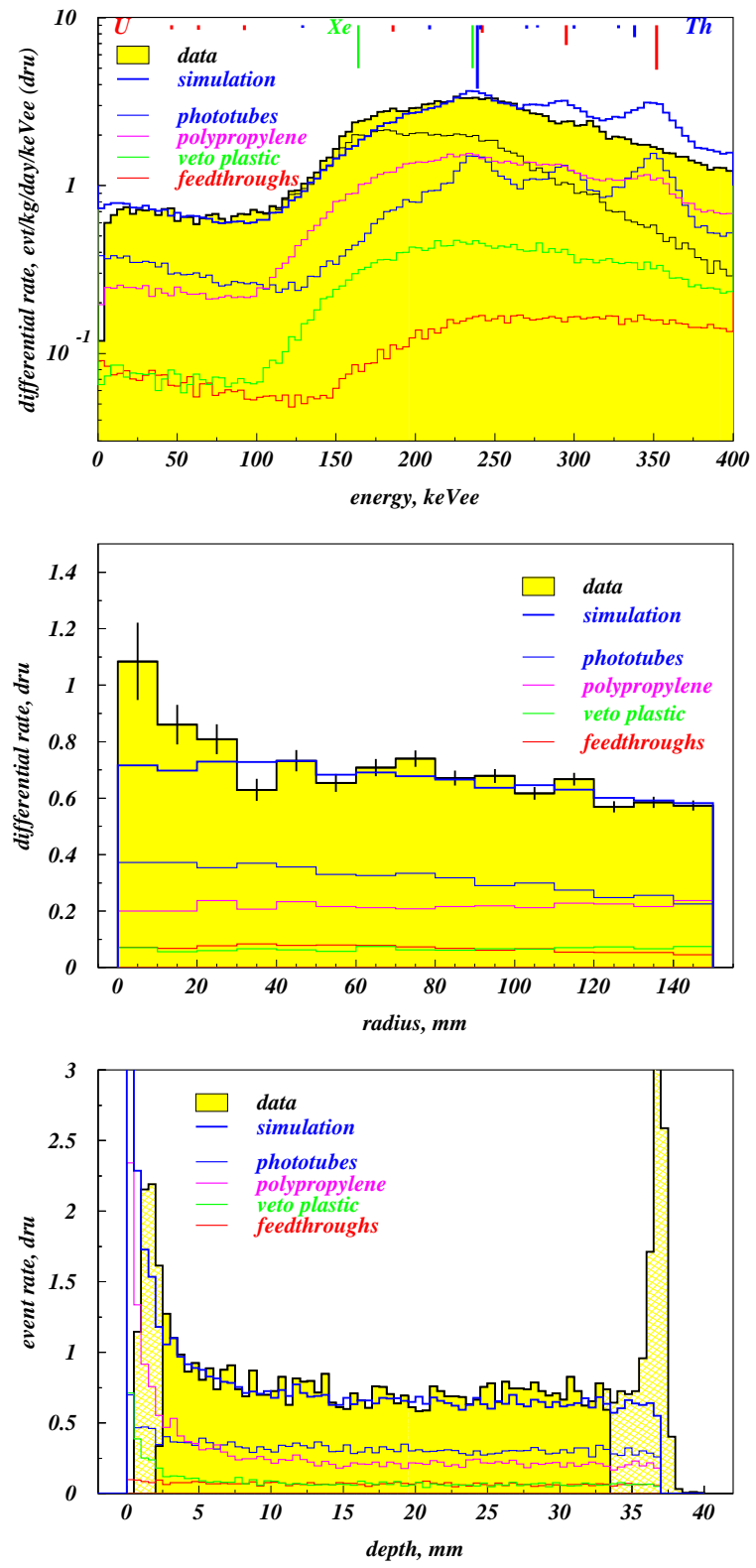

Figure 2: Electron recoil background in ZEPLIN-III. Data are shown in yellow and simulated components are summed by the solid blue line, with individual contributions ordered by importance at low energy. Top: energy spectrum in fiducial volume, showing good agreement below $\sim 150 \mathrm{keVee}$, the dynamic range of the dataset (the thin black histogram retains only unsaturated events); U/Th and Xe activation $\gamma$-rays are indicated. Middle: radial distribution below 100 keVee, confirming very good agreement beyond the central channel. Bottom: depth distribution for fiducial region (bright yellow) and full depth (hatched yellow); contamination of the cathode grid $(37 \mathrm{~mm})$ with radon progeny left over from the first run is clearly visible (but easily fiducialised away). 
single S1 and S2 pulses are selected, which allows three-dimensional reconstruction of the interaction point. A $6.5 \mathrm{~kg}$ fiducial region $(150 \mathrm{~mm}$ radius, $32 \mathrm{~mm}$ deep) is retained for analysis, with no significant quality cuts applied. The energy variable used is a linear combination of S1 and S2 energy estimators which exploits the anti-correlation between these two response channels (ionisation electrons may contribute either to S2, if extracted from the track, or to $\mathrm{S} 1$, via recombination luminescence). This allows for a better measurement of energy than either channel independently. The dynamic range of the dataset is limited, owing mainly to the 8bit resolution of the digitisers. Although a dualrange acquisition system is used, the large $\mathrm{S} 2$ gain means that signal truncation becomes significant above $\sim 150$ keVee in the WIMP-search dataset.

The measured energy and spatial distributions are shown in Figure 2 - along with a componentlevel breakdown from simulated data calculated as described below. These contributions are also listed in Table I. The measured low-energy background is $0.75 \pm 0.05$ events $/ \mathrm{kg} /$ day $/$ keVee (hereafter 'dru', for 'differential rate unit'). Simulated and measured spectra are in good agreement up to $\sim 150 \mathrm{keV}$ and even beyond, despite the xenon activation which is still prominent in this dataset. This represents a $\sim 20$-fold improvement over the first run. The simulation sum predicts $0.85 \pm 0.05 \mathrm{dru}$ at low energy; as discussed further ahead, this is based on measured contamination only, i.e. excluding items for which contamination levels could not be detected. Aside from this selection, there is no scaling of the simulation results in that figure.

The spatial distributions are also in good agreement, with two exceptions. The radial trend matches, within errors, over most of the fiducial volume, but the rate is slightly higher than predicted within the radius corresponding to the central photomultiplier $(26.5 \mathrm{~mm})$; this is explained by higher than average contamination in this device (but still within expected batch variability). The vertical distribution is also reproduced closely, except for the spike observed near $37 \mathrm{~mm}$, the depth of the cathode wire grid. This is due to a previous contamination with radon arising in the xenon purification getters used prior to the first run. We subsequently found these to emanate $\sim 1$ atom/s, leading to significant implantation of ${ }^{210} \mathrm{~Pb}$ in some detector surfaces. Notwithstanding, both energy and spatial distributions of electron recoils are well reproduced by the simulation within the fiducial volume.

\subsection{Plating of radon progeny on the cathode}

Although other primordial decay chains also include radon isotopes, ${ }^{222} \mathrm{Rn}\left({ }^{238} \mathrm{U}\right.$ chain $)$ is the longest lived $\left(\mathrm{T}_{1 / 2}=3.824\right.$ days $)$. The issue of getter emanation had been previously identified in ZEPLIN-II [10], which relied on continuous gas recirculation through the same getter model (SAES PS11-MC500). Upon interruption of purification, the decay of the $\alpha$ activity was measured at $\mathrm{T}_{1 / 2}=3.83 \pm 0.1$ days, supporting a ${ }^{222} \mathrm{Rn}$ contamination [17]. By then the same getters had been used to purify ZEPLIN-III xenon before the first run, which led to internal plating with ${ }^{210} \mathrm{~Pb}$. This did not pose a problem then, and we opted not to etch it away during the upgrade; we replaced the getter with a new model (SAES PS4-MT3). The effect of internal radon decay can be understood with reference to the chain sequence:

$$
\begin{aligned}
& { }^{222} \mathrm{Rn}(\alpha, 3.8 \mathrm{~d}) \rightarrow{ }^{218} \mathrm{Po}(\alpha, 3.1 \mathrm{~m}) \rightarrow{ }^{214} \mathrm{~Pb}\left(\beta^{-}, 27 \mathrm{~m}\right) \rightarrow \\
& { }^{214} \mathrm{Bi}\left(\beta^{-}, 20 \mathrm{~m}\right) \rightarrow{ }^{214} \mathrm{Po}(\alpha, 164 \mu \mathrm{s}) \rightarrow{ }^{\mathbf{2 1 0}} \mathrm{Pb}\left(\beta^{-}, \mathbf{2 2 y}\right) \rightarrow \\
& { }^{210} \mathrm{Bi}\left(\beta^{-}, 5 \mathrm{~d}\right) \rightarrow{ }^{210} \mathrm{Po}(\alpha, 138 \mathrm{~d}) \rightarrow{ }^{206} \mathrm{~Pb}(\text { stable })
\end{aligned}
$$

Once washed into the target the radon mixes uniformly in the LXe. The first two $\alpha$ decays produce negatively charged atoms which promptly lose excess electrons (the $\alpha$-particle drifts to the cathode where it reduces to $\mathrm{He}$ ). The next two $\beta^{-}$decays create ${ }^{214} \mathrm{Bi}^{+}$and ${ }^{214} \mathrm{Po}^{+}$ions which cannot transfer their ionisation state to the rare gas and thus drift to the cathode. Finally, the $\alpha$ decay of ${ }^{214} \mathrm{Po}$ implants ${ }^{210} \mathrm{~Pb}\left(\mathrm{~T}_{1 / 2}=22.3 \mathrm{yr}\right)$ within the wire grid, which bottlenecks the chain. This ${ }^{210} \mathrm{~Pb}$ activity is still present on the cathode in the second run and has remained approximately stable at $19.4 \pm 0.3$ decays/day over several months (per each of ${ }^{210} \mathrm{~Pb}$, ${ }^{210} \mathrm{Bi}$ and ${ }^{210} \mathrm{Po}$, as measured by the rate of ${ }^{210} \mathrm{Po}$ nuclear recoils).

The electron-recoil background arising in the fiducial volume from this contamination is insignificant in the context of the overall radioactivity budget (and was not included in the simulation); the same is true of nuclear recoils from the $(\alpha, n)$ reaction from ${ }^{210} \mathrm{Po}$ on the stainless steel wires. In fact, these populations can be useful as stable sources of ionisation; they allow, for example, the ionisation yield for the deepest events to be monitored as a function of time. The Bateman decay equations for the ${ }^{210} \mathrm{~Pb}$ sub-chain indicate that the ratios of ${ }^{210} \mathrm{~Pb},{ }^{210} \mathrm{Bi}$ and ${ }^{210} \mathrm{Po}$ become constant after $\sim 2$ years (which is approximately the period 
Table 1: Component-level analysis of electron recoil background and nuclear recoils from neutron elastic scattering in ZEPLIN-III. The sum of simulated contributions (excluding components without measured contamination, indicated in brackets) is labelled 'SSR total'. The additional rejection efficiency provided by the veto is indicated: 'ptag' for prompt tags, which remove $\gamma$-rays, and 'dtag' for delayed tags, which reject neutrons [5] (weighted averages are indicated under 'SSR total'; these agree with the measured tagging efficiencies). After veto tagging, the neutron event expectation in a 1 year long dataset with typical signal acceptance is some 10 times lower $(\simeq 0.4$ events).

\begin{tabular}{l|c|cc|cc}
\hline \hline Material & mass, kg & e-recoil, dru & ptag & n/year & dtag \\
\hline Krypton-85 & - & 0.007 & $\sim 0$ & - & - \\
Ceramic feedthroughs & 0.9 & 0.08 & 0.30 & 1.35 & 0.58 \\
Photomultipliers & 4.2 & 0.40 & 0.26 & 0.74 & 0.58 \\
Rock (halite) & - & $\sim 0$ & $\sim 0$ & 0.53 & 0.58 \\
Polypropylene shield & 1,266 & 0.25 & 0.04 & 0.10 & 0.58 \\
Scintillator modules & 1,057 & 0.09 & $\sim 1$ & 0.03 & $\sim 1$ \\
Copperware & $\sim 400$ & $(<0.1)$ & 0.10 & $(<0.15)$ & 0.58 \\
Lead castle & $\sim 60,000$ & 0.01 & 0.54 & $\sim 0$ & 0.58 \\
Radon-222 & $1 \mathrm{~m}^{3}$ & 0.03 & 0.19 & $\sim 0$ & - \\
Muon-induced & - & - & & $\sim 0.3$ & $\sim 1$ \\
\hline SSR total & & $0.86 \pm 0.05$ & 0.28 & $3.05 \pm 0.5$ & 0.58 \\
SSR data & & $0.75 \pm 0.05$ & 0.28 & $\mathrm{n} / \mathrm{a}$ & - \\
\hline (FSR [6] & & $14.5 \pm 0.5$ & - & $(36 \pm 18)^{*}$ & $-)$ \\
\hline \hline
\end{tabular}

$\dagger$ events/kg/day/keVee at $10 \mathrm{keVee}$

$\ddagger$ single scatters in $2,370 \mathrm{~kg} \cdot$ days over $5-50 \mathrm{keVnr}$ (unity detection efficiency)

* FSR dataset expectation was $1.2 \pm 0.6$ events in WIMP search box (net $128 \mathrm{~kg}$.days)

elapsed between the start of the two runs); when this so-called transient equilibrium is reached, the relative spectrum of decay products becomes constant, and so does the mean ionisation yield per event. Monitoring the S2/S1 ratio for these lowenergy events allows, for example, the accuracy of the electron lifetime correction to be checked.

\subsection{Photomultiplier $\gamma$-rays}

The original phototubes (2-inch ETEL D730Q) were purchased a decade ago and had relatively high contamination levels (250 ppb U, $290 \mathrm{ppb}$ Th and $1350 \mathrm{ppm} \mathrm{K}$ ), releasing $1,400 \mathrm{mBq}$ per unit in $\gamma$-ray activity. This dominated by far the background budget in the first run $(14.5 \mathrm{dru})$. For this reason a second run of the experiment with upgraded devices was planned from the outset. A low-background product (D766Q) was developed by ETEL in collaboration with the project to enable pin-by-pin compatibility with the existing array. All materials and sub-components were radioassayed with HPGe and/or ICP-MS. The best phototubes have $35 \mathrm{mBq}$ in $\gamma$-rays, which was achieved through a complete redesign of the device. Most of this activity is concentrated at the rear of the envelope, away from the fiducial volume. Simulations of background contribution to ZEPLIN-III take the spatial distribution of activity into consideration. A value of $0.40 \mathrm{dru}$ is predicted from internal $\gamma$ rays, which represents a 40 -fold improvement over the previous array and makes this component only marginally dominant. The equivalent simulations for the first run reproduced data well, which gives added confidence in this calculation. Backgrounds arising from the $\beta^{-}$activity internal to the phototubes, which are often neglected in this type of study, are discussed in Section 3.1 .

\subsection{Krypton-85 and other intrinsic backgrounds}

Several LXe-intrinsic backgrounds can affect this and similar experiments. ${ }^{210} \mathrm{~Pb}$ from ${ }^{238} \mathrm{U}$ and ${ }^{222} \mathrm{Rn}$ chains and ${ }^{228} \mathrm{Ra}$ from the ${ }^{232} \mathrm{Th}$ chain, for example, undergo low-energy $\beta^{-}$decay occasionally lacking significant $\mathrm{x}$ - and $\gamma$-rays to help flag these events ('naked betas'). Tritium $\left(\mathrm{T}_{1 / 2}=12.3 \mathrm{yr}\right.$, $\left.\beta_{\max }=19 \mathrm{keV}\right)$ is produced cosmogenically and, on some accounts, it could generate as much as $\sim 1 \mathrm{dru}$ if the xenon is stored on the surface for long periods [18]. However, it is reasonable to assume that these contaminants can be removed effectively during xenon purification and, so long as radon emanation into the detector can be controlled, they 
should not come to dominate the background at low energy. We see no evidence for a substantial presence of these in the second run of ZEPLIN-III.

More significant is the threat posed by ${ }^{85} \mathrm{Kr}$, long recognised as a serious background for WIMP searches. ${ }^{85} \mathrm{Kr}$ is an anthropogenic $\beta^{-}$emitter $\left(\mathrm{T}_{1 / 2}=10.76 \mathrm{yr}, \beta_{\max }=687 \mathrm{keV}\right)$; its presence in the atmosphere is mainly due to the reprocessing of spent nuclear fuel. The pre-nuclear ${ }^{85} \mathrm{Kr} / \mathrm{Kr}$ ratio was as low as $3 \times 10^{-18}$ in the early 1950 s, but the present-day ratio is $\sim 1 \times 10^{-11}$ [19].

The xenon used in ZEPLIN-III was sourced in the 1970s from an underground origin. An accurate measurement of its ${ }^{85} \mathrm{Kr}$ content was obtained in 1997 from a $5.9 \mathrm{~g}$ sample [20], from which we derive an age-corrected ${ }^{85} \mathrm{Kr} / \mathrm{Kr}$ ratio of $1.5 \times 10^{-12}$. The overall $\mathrm{Kr}$ content was subsequently reduced by cryogenic distillation to $\sim 50 \mathrm{ppb} \mathrm{Kr}$ (by weight), corresponding to $0.2 \mathrm{dru}$ at low energy in the xenon target. This was, however, a conservative estimate and the present data suggest much lower contamination, which can be confirmed independently.

A minor $\beta^{-}$decay $\left(0.434 \%, \beta_{\max }=173 \mathrm{keV}\right)$ is accompanied by a $514 \mathrm{keV} \gamma$-ray from the ${ }^{85} \mathrm{Rb} 9 / 2^{+}$ level $\left(\mathrm{T}_{1 / 2}=1.015 \mu \mathrm{s}\right)$ [21]. This allows a $\beta-\gamma$ delayed coincidence analysis, with the $\gamma$-ray searched in the veto scintillator or in the LXe target. A 6month dataset revealed $\sim 9$ such coincidences in the target, conforming to the expected time delay distribution. This translates to $4 \times 10^{-21}{ }^{85} \mathrm{Kr} / \mathrm{Xe}$ ratio (in present-day xenon this would represent a very low $150 \mathrm{ppt} \mathrm{Kr}$ ). The decay rate is $0.007 \pm 0.002 \mathrm{dru}$, which is insignificant in ZEPLIN-III.

\subsection{Ceramics and copperware}

Along with the photomultipliers, two materials could make up a significant fraction of the radioactivity budget of the experiment from the outset: the $\sim 400 \mathrm{~kg}$ of copper (from which all structural elements, vessels, fastening hardware parts, etc., were manufactured) and ceramic feedthroughs. Other items were screened and their locations chosen to minimise their impact: stainless steel parts (e.g. vacuum ports), indium seals, $\mathrm{LN}_{2}$ in the 30 -litre reservoir.

For copper (OFHC C103), conservative upper limits of $0.5 \mathrm{ppb}$ for $\mathrm{U} / \mathrm{Th}$ contamination were initially adopted, although lower values existed in our database for similar material [15]. Simulation of this contribution indicated an electron recoil background of 0.35 dru at low energy. However, the measured energy and spatial distributions show no evidence for such a significant rate, and we revised the upper limit down to $0.1 \mathrm{dru}$ for the primordial radioactivity.

Cosmogenic activation also produces several radio-isotopes, of which ${ }^{60} \mathrm{Co}\left(\mathrm{T}_{1 / 2}=5.3 \mathrm{yr}\right)$ will retain the highest activity a few years after exposure to atmospheric muons ceases. We adopt a production rate for ${ }^{60} \mathrm{Co}$ in natural copper of $\sim 50$ atoms $/ \mathrm{kg} /$ day [18]. Since the exact exposure time on the surface is uncertain we assume secular equilibrium, with a subsequent cool-off period of 4 years (preceding the second run after deployment underground). Simulation of the resulting $\gamma$-ray activity within the copperware produces a negligible $0.01 \mathrm{dru}$ in the fiducial volume.

There are nearly 100 ceramic feedthroughs for signal and high voltage connections, weighing $900 \mathrm{~g}$ in total; these are distributed among the xenon chamber baseplate and the vacuum vessel flange. They are alumina based and their average contamination was measured at $105 \mathrm{ppb}$ U, $270 \mathrm{ppb}$ Th and $880 \mathrm{ppm} \mathrm{K}$. The $\gamma$-ray background is not severe (0.08 dru), but they dominate the neutron background in the detector as discussed later.

\subsection{Polypropylene shield and veto detector}

The ZEPLIN-III veto consists of a tight-fitting polypropylene shield enveloping the main instrument, itself surrounded by 52 plastic scintillator modules forming barrel and roof sections. The gadolinium-loaded hydrocarbon moderates and captures internal neutrons very efficiently; the Gd capture $\gamma$-rays are detected by the scintillators. The neutron detection efficiency (delayed tagging in a $70 \mu$ s window) is $58 \%$; internal $\gamma$-rays are detected with an average $28 \%$ efficiency (prompt tagging in $\pm 0.2 \mu$ s window). The prompt tag adds a further $2 \%$ to the neutron efficiency. A detailed discussion of the hardware and physics performance is given elsewhere [4, 5]; there, we analyse the backgrounds contributed to the WIMP target (a prime design consideration) as well as background measurements in the veto detector itself. A tonne of scintillator within the lead castle provides confirmation of the local radiation environment and we find that the veto measurements agree with the background measured within ZEPLIN-III itself.

The readout (photomultipliers, electronics, cabling, etc.) are external to the hydrocarbon shielding to mitigate neutrons, which is achieved very successfully. These also have a negligible contribution 
to the $\gamma$-ray background in the LXe target. However, owing to their large mass, the polypropylene and the plastic scintillator do contribute appreciably to the ZEPLIN-III budget.Contamination levels have been measured for the polypropylene (1 ppb $\mathrm{U}, 1 \mathrm{ppb}$ Th and $5 \mathrm{ppm} \mathrm{K}$ ) and the plastic scintillator (0.2 ppb U, $0.1 \mathrm{ppb}$ Th and $0.2 \mathrm{ppm} \mathrm{K}$ ). These values are close to the sensitivity of the HPGe and ICP-MS measurements of those samples, but this radiological contamination is entirely consistent with the interaction rate recorded in the veto itself. Furthermore, these have distinct energy and spatial distributions in ZEPLIN-III, as shown in Figure 2, which confirm these contributions: the energy spectra are generally softer and they increase the event rate especially at the top of the xenon target, in contrast with other dominant components, such as the phototubes and ceramics, which are located below the xenon volume.

We note that radioactivity in the plastic scintillator does not contribute to the overall background since the $Q$-value of the radioactive decay, most of which is deposited locally, is nearly always sufficient to trigger the veto (even if the highest energy $\gamma$-rays are able to escape). On the other hand, the polypropylene generates $0.25 \mathrm{dru}$ in the xenon target and has a low veto probability of $4 \%$.

\section{7. $\gamma$-rays inside lead castle}

The $\gamma$-ray flux inside the empty ZEPLIN-III castle includes external contributions from the laboratory walls, natural as well as cosmogenic activity in the shield, air-borne radon and its metallic progeny plated out on the inner castle walls. $\gamma$ rays from Boulby rock (halite) are attenuated by a factor of $\sim 10^{5}$ by the $20-\mathrm{cm}$ thick lead shield [22] and are insignificant as a background in the WIMP target. The activity of the ${ }^{222} \mathrm{Rn}$ chain measured with a commercial monitor inside the empty castle was $2.4 \mathrm{~Bq} / \mathrm{m}^{3}$, which is typical at Boulby. Simulation of $\gamma$-rays from this activity leads to $0.03 \mathrm{dru}$ in ZEPLIN-III. Further in situ measurements with a HPGe detector place an upper limit on the background from the castle (primordial and cosmogenic activity in the bulk shield and inner walls). The castle walls and roof are made from lead smelted into thin stainless steel containers to make 1.2tonne chevron-shaped blocks; the lead had been underground for some two decades and is known to have low ${ }^{210} \mathrm{~Pb}$ content (it was used to shield previous dark matter experiments at Boulby). The shield flooring is OFHC copper, which was moved underground over a decade ago as 'new copper'. The HPGe spectra show only a vestigial amount of ${ }^{60} \mathrm{Co}$. The combined contribution of the shield to ZEPLIN-III is $\sim 0.01 \mathrm{dru}$.

\section{Rarer types of electron recoil event}

\section{1. $\beta$ - and $\gamma$-induced photomultiplier backgrounds}

A significant amount of potassium is involved in sensitising internal photomultiplier surfaces, namely the bialkali photocathodes. The ${ }^{40} \mathrm{~K}$ thus located close to the fiducial volume can generate a response in the detector in several ways, with the $1,461 \mathrm{keV}$ EC $\gamma$-rays being perhaps the most obvious. However, the $89 \%$ branching ratio $\beta^{-}$decay $\left(\beta_{\max }=1,311 \mathrm{keV}\right)$ can be as problematic (this represents $30 \mathrm{mBq}$ in the $\mathrm{D} 766 \mathrm{Q})$. VUV-sensitive phototubes have thin quartz windows $(\sim 1 \mathrm{~mm})$, allowing some internal $\beta$-particles to reach the LXe. Others will generate bremsstrahlung photons in the quartz. Cherenkov photons are also produced in the quartz and can be detected in coincidence with the prompt scintillation. Other luminescence processes arise internally in the phototubes, such as 'dynode glow' from electron impact, which is signal coincident. Besides contributing some $10-20 \%$ to the detector trigger rate, these $\beta$-induced events can lead to backgrounds which are harder to discriminate than single scatters in the fiducial volume. These might combine, for example, bremsstrahlung photons interacting in the main volume with Cherenkov light from the quartz, thus decreasing the observed $\mathrm{S} 2 / \mathrm{S} 1$ ratio of the fiducial interaction. Cherenkov emission from Compton electrons created by $\gamma$-ray backgrounds may be even more damaging as fewer photoelectrons may be involved in this instance.

Figure 3 illustrates some of these processes; it shows Monte Carlo data for ${ }^{40} \mathrm{~K}$ decays generated internally in the central phototube in ZEPLIN-III. The quartz windows are curved and $<1 \mathrm{~mm}$ thick. The blue histogram (cumulative) represents energy deposits everywhere in LXe whereas the red histogram refers to the active region above the cathode only (mainly $\gamma$-rays). Transmitted $\beta$-particles and bremsstrahlung in the quartz window clearly dominate at low energies for interactions just above the phototube. In the fiducial volume, some $10 \mathrm{~mm}$ away, these contributions become negligible.

As mentioned above, Cherenkov light generated inside the quartz may also contribute to S1, yielding potentially tens of photoelectrons. For example, a $1 \mathrm{MeV}$ electron - e.g. a ${ }^{40} \mathrm{~K} \beta$-particle or a 


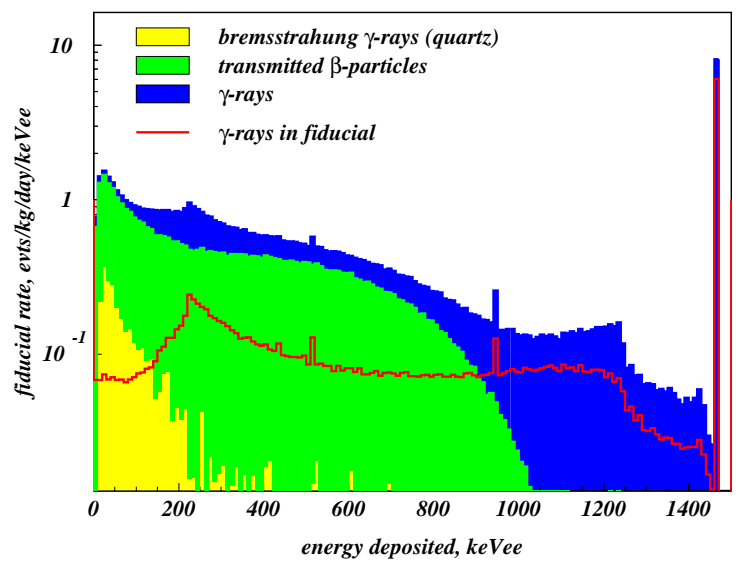

Figure 3: Energy spectrum in LXe from simulated ${ }^{40} \mathrm{~K}$ decays internal to central photomultiplier; curves are scaled $(\times 31)$ for correct fiducial rate from this contribution. The solid histograms are cumulative and represent interactions above the phototube window; these include bremsstrahlung photons generated in the quartz, transmitted $\beta$-particles and non-bremsstrahlung $\gamma$-rays. The spectrum above the cathode is shown in red, mostly due to $1,461 \mathrm{keV} \gamma$-rays.

Compton electron from the $1,461 \mathrm{MeV} \gamma$-ray - produces $\sim 100$ photons $/ \mathrm{mm}$ of quartz within the spectral response of these photomultipliers; some generate photoelectrons even without leaving the window, while others suffer total internal reflection at the liquid surface and strike the array again; therefore, the photoelectron yield can be significantly higher than suggested by the nominal QE. This optical signal can both increase the size of a timecoincident scintillation pulse as well as reduce its apparent time constant, threatening single-phase (scintillation only) as well as two-phase detectors. We see evidence of Cherenkov emission in ZEPLINIII. This is illustrated in Figure 4, where the pulse timing parameter $\tau$, which measures the mean arrival time of the $\mathrm{S} 1$ signal, is shown for fiducial scintillation pulses and for sub-cathode events (no S2) with the same range of apparent energies (30-60 keVee). The latter are mainly due to $\beta$-particles transmitted through the phototube windows - but a faster population is also clearly seen which we attribute to Cherenkov emission. We can rule out an explanation based on nuclear recoils on the underside of the cathode grid, which would indeed be faster than electron recoils and yield no ionisation, by comparing with the corresponding timing spectrum for the upper grid surface. We conclude that pulses in this population are indeed faster than nuclear recoil scintillation. More extreme examples can be identified if signals triggering in a single channel are included (such as the blue pulse shown in inset), but in this instance it is harder to prove an optical, rather than electrical, origin.

Such Cherenkov processes can be dangerous if associated with low-energy electron recoils in the fiducial volume. Besides Compton electrons generated by background $\gamma$-rays, this could involve, more generally, $\beta^{-}$decays with coincident $\gamma$-rays (many examples exist in the $\mathrm{U}$ and Th chains). In ZEPLINIII, the timing distribution of fiducial events with higher than average scintillation (lower S2/S1 ratio) at energies above 30 keVee does not correlate with shorter than average pulses; rather, it is fully compatible with that of electron recoils - although this conclusion stems from a relatively small exposure. In any case, this effect should be carefully assessed in future noble liquid instruments where scintillation is detected by photomultipliers.

For this and other reasons optical cross-talk between phototubes must be avoided, so that these effects remain localised to a single channel and are therefore easier to identify. It is also important to reduce the amount of LXe allowed around their envelopes to minimise scintillation from internal $\beta$ particles, and to prevent those photons from reaching the photocathode either by external or internal paths. The copper structures which house the ZEPLIN-III phototubes allow a thicker LXe layer around their envelopes than with the original ones, since the upgrade photomultipliers are slightly narrower. However, there is no significant direct path into the main volume and the quartz envelopes were metallised to prevent light from reaching the photocathode directly.

\subsection{Multiple $\gamma$-ray interactions: MSSI events}

Multiple interactions where one vertex occurs in a detector region which is optically coupled to the phototube array but from which no charge can be extracted can lead to a challenging background. Multiple-scintillation single-ionisation (MSSI) events produce lower S2/S1 ratios than single fiducial scatters since the multiple S1 pulses are time-coincident but there is only one S2. This was identified early on as a background mechanism in two-phase detectors. In ZEPLIN-III an initial assessment during construction showed that efficient rejection based on S1 light pattern and S1-S2 vertex consistency would be necessary [2]. In the first 


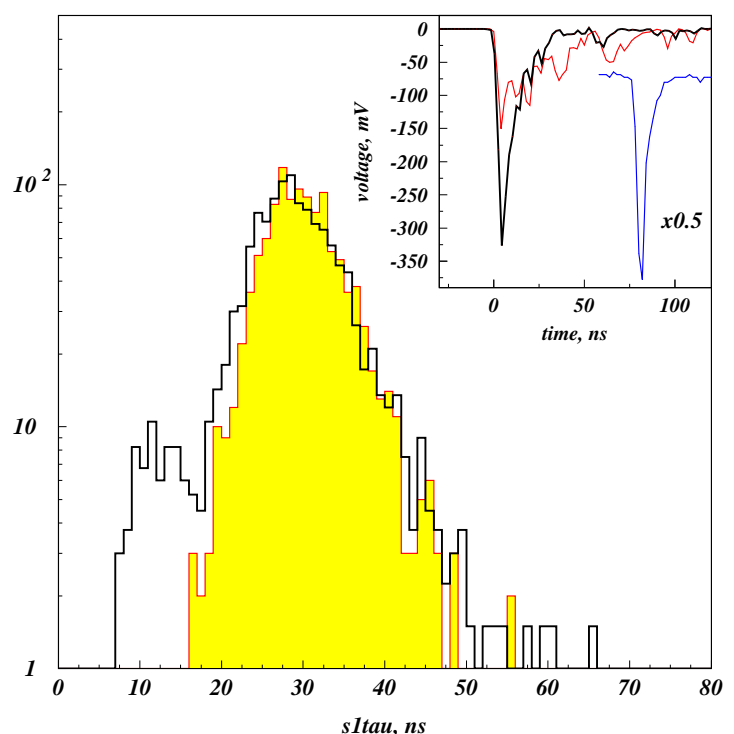

Figure 4: Distribution of photoelectron mean arrival times $(\tau)$ in S1-like pulses for fiducial interactions (yellow histogram) and for S1-only events occurring between the photomultiplier windows and the cathode grid. The apparent energies are 30-60 keVee for both histograms. A population of fast pulses is clearly visible, which we attribute to Cherenkov photons generated in the phototube windows by internal $\beta^{-}$emission. Inset: array time response for three events with 30 keVee reconstructed energy (approximately 55 photoelectrons); the red trace is a fiducial event with $\tau=35 \mathrm{~ns}$; the black trace, probably a scintillation event contaminated by significant Cherenkov light, is chosen from the black histogram with $\tau=13 \mathrm{~ns}$. The pulse in blue has $\tau=7 \mathrm{~ns}$ and occurs in only one channel (the other cases both trigger 7 channels).

run this event topology became perhaps the most challenging, in particular because these populations cannot be reproduced accurately with calibration point sources.

A detailed calculation is difficult when the light collection for the dead vertex is not known precisely. In ZEPLIN-III, this is the case in two regions which yield no charge: i) the reverse field region below the cathode grid, where light collection is high but variable due to the proximity to the photomultiplier windows; ii) the peripheral region near the side walls, where the light collection is low and also uncertain. In any case, it is instructive to assess what effect these events will have on discrimination if not successfully rejected. Consider two $\gamma$-ray interactions, one in the fiducial region and one in a dead region, which we assume to have equal light collection and a constant spectrum of en- ergy deposited (as suggested by the Klein-Nishina formula for low-angle scattering of high energy $\gamma$ rays). The energy is reconstructed from the coincident S1 components, but there is only one S2. The number of such double scatters adding up to energy $E$ is $P(E) d E=P_{0}^{2} E d E$, where $P_{0}$ is the single-scatter probability per unit energy. So, this effect becomes more frequent with increasing reconstructed energy, as might be expected. The discrimination parameter, $\mathrm{S} 2 / \mathrm{S} 1$, will suffer most when the 'good' vertex produces the smallest detectable S2 signal $\left(E_{t h}\right)$ and $\mathrm{S} 1$ takes its apparent energy from the dead vertex only $\left(\mathrm{S} 2 / \mathrm{S} 1>E_{t h} / E\right)$. However, the $(x, y)$ positions recovered from $\mathrm{S} 1$ and $\mathrm{S} 2$ independently are likely to be inconsistent for these events, leading to their probable rejection. Therefore, the good S1 vertex must produce a minimum viable signature and, in practice, $E_{t h} \sim 2.5 \mathrm{keVee}$, the S1 energy threshold. This trend is indeed observed in our data before these events are removed by the vertex reconstruction algorithm. A similar calculation can be done for neutron interactions. Assuming, very approximately, an exponential recoil spectrum with characteristic energy $E_{0}$, then the spectrum of double-vertex events is $P(E) d E \propto E \exp \left(-E / E_{0}\right) d E$ (i.e. the relative number of such events still increases with $E$ ).

\section{Radioactivity neutrons}

The agreement between data and simulations obtained for the spectrum of electron recoils at low energy confirms the radiological contaminations adopted for each component; these were subsequently used to derive neutron production rates in those materials. Muon-induced showers, in particular photo-production in electromagnetic cascades, are the only noteworthy source of non-radioactivity neutrons. This contribution is not dominant in a detector of the size of ZEPLIN-III 23, 24] and, in addition, these neutrons are vetoed effectively. The dominant sources of fast neutrons are, therefore, spontaneous fission (mostly of ${ }^{238} \mathrm{U}$ ) and $(\alpha, \mathrm{n})$ reactions arising from the $\mathrm{U}$ and $\mathrm{Th}$ chains

Absolute neutron spectra were calculated with SOURCES-4A and -4C [25, 26] with $(\alpha, n)$ crosssections extended to $10 \mathrm{MeV}$ as described in

\footnotetext{
${ }^{2} \mathrm{An} \alpha$ activity of $10 \mathrm{~Bq}$ is present in the polypropylene due to ${ }^{152} \mathrm{Gd}$, but this is not a significant source of neutrons $\left(E_{\alpha}=2.8 \mathrm{MeV}\right)$.
} 
Ref. 27] and complemented with EMPIRE2.19 28] cross-sections and branching ratios for transitions to excited states [29]. The $(\alpha, n)$ reaction dominates in light materials, producing, e.g., nearly all neutrons emitted by the Boulby rock (considered pure $\mathrm{NaCl}$ here), very few in copper $(<10 \%)$ and practically none in lead. Yields in pure elemental materials are generally in good agreement with experimental data, but it should be noted that the exact composition, uniformity and granularity of mixtures in detector components is a cause of uncertainty due to the very short range of $\alpha$-particles.

Primary neutron spectra are generated isotropically from an appropriate position in the GEANT4 simulation geometry and tracked to the LXe target. In the case of the photomultipliers this is done at sub-component level, since some materials are further away from the fiducial volume than others. Each photomultiplier emits just over 2 neutrons per year; this is dominated by a small amount of borosilicate glass still present in this model; although there is scope for further improvement, this value represents a 50-fold reduction relative to those used in the first phase of the experiment.

The most challenging calculation is the contribution from the laboratory rock. It is computationally intensive and requires accurate modelling of the shield: in spite of the high overall attenuation factor of $\sim 10^{5}$, any gaps for pipework, cabling and alignment tolerances need to be taken into account. To ensure an accurate result a detailed CAD solid model of the veto and shield was imported into the GEANT4 model. The Boulby rock has $65 \mathrm{ppb} \mathrm{U}$ and $130 \mathrm{ppb}$ Th assumed in secular equilibrium [30]. Neutrons are generated in the rock to a depth of $3 \mathrm{~m}$ and tallied initially on a test surface in the laboratory. The simulated integral flux is adjusted at this stage to measurements at Boulby 31], including re-entrant (i.e. backscattered) neutrons. The differential spectrum is released with a cosine bias from a generator sphere containing the experiment to generate an isotropic and uniform flux within it.

Yearly rates of single scatters in 5-50 keVnr in the fiducial volume and the component-level breakdown are given in Table I and shown in Figure 5 The total of 3.05 events per year is dominated by the ceramic feedthroughs $(44 \%)$, followed by the photomultipliers and rock neutrons. We note that the background expectation predicted for a oneyear dataset with realistic signal acceptance is considerably lower; typical signal detection efficiencies (including 90\% operational duty cycle, 50\% accep- tance in $\mathrm{S} 2 / \mathrm{S} 1$ discrimination parameter, a further $25 \%$ loss from software selection cuts), bring this down to $1.0 \mathrm{n} /$ year; the rate in anti-coincidence with the veto is $60 \%$ lower, at $0.4 \mathrm{n} /$ year. Finally, the detection threshold is likely to be a little higher than $5 \mathrm{keVnr}$, so a value just below $0.3 \mathrm{n} /$ year is expected. This is very close to the original goal of the experiment. The veto-coincident data analysed so far contains zero delayed coincidences (dtag) within the signal acceptance region, but this constrains the latter event rate only to $<3 \mathrm{n} /$ year at $90 \% \mathrm{CL}$ (note that vetoed events have not been subject to a blind analysis since they contains no information on the presence of a signal).

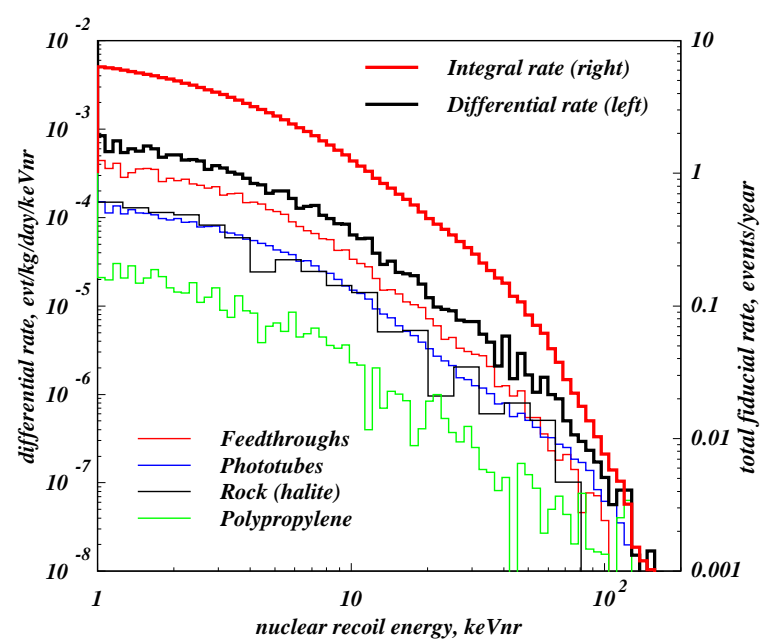

Figure 5: Simulated differential and integral rates of neutron single elastic scattering in the fiducial volume of ZEPLIN-III.

The event rates discussed above are for single scatters in the $6.5 \mathrm{~kg}$ fiducial volume with no additional interactions (elastic or otherwise) in the $12.5 \mathrm{~kg}$ active volume. Where more than one elastic scatter occurs, the low $\mathrm{S} 2 / \mathrm{S} 1$ ratios (and short scintillation time constant) characteristic of nuclear recoils are essentially preserved. Although the event can still be rejected from the multiple S2 pulses, it is often useful to establish the distribution of scattering multiplicity. This may provide additional constraints to the expected rate of single scatters, which are an irreducible background for WIMP searches. In ZEPLIN-III, some $50 \%$ of events with energies reconstructed in the $5-50 \mathrm{keVnr}$ region are single interactions. The mean scattering length varies quite significantly with neutron energy, but it is much less sensitive as a function of recoil en- 
ergy $(\lambda \sim 30 \mathrm{~cm})$. For systems much larger than this value, self-shielding can render the fraction of single scatters very small. Multiple scatters can be identified by additional $\mathrm{S} 2$ pulses in two-phase systems (although they will become MSSI events if the second vertex yields no charge). However, the timeof-flight for radioactivity neutrons should not be neglected as a valuable discriminant. The mean time between scatters (above threshold) is only $\sim 15 \mathrm{~ns}$ in ZEPLIN-III, but this effect can be readily seen in the structure of S1 pulses during neutron calibration, which points to its usefulness in large singlephase detectors.

\section{Discussion}

In this study we analysed electron and nuclear recoil backgrounds of radiological origin in the ZEPLIN-III experiment now operating in its second phase at Boulby. Energy spectra and spatial distributions are presented for electron recoils in the fiducial volume. The low energy rate is $0.75 \pm 0.05 \mathrm{evt} / \mathrm{kg} /$ day $/ \mathrm{keVee}$, which represents a 20 -fold improvement relative to pre-upgrade levels. A further $28 \%$ reduction is possible due to the new veto detector installed around the WIMP target. Significantly, these vetoed events can be studied without compromising a blind analysis of the signal region.

The electronic background achieved in phase II meets the original goal of the ZEPLIN-III programme. It implies a leakage of $2-3$ events/year for a discrimination power of 1:7,800 (the value achieved in the first science run). Whether this can be matched in the second run depends on the optical performance of the photomultipliers, which unfortunately is not ideal.

A long campaign of component-level radioassays (and Monte Carlo simulations to determine their contribution to the radioactivity budget) led to good matches to the measured distributions. We confirmed that the photomultipliers and the polypropylene shield dominate, while copper and ${ }^{85} \mathrm{Kr}$, identified early on as possible concerns, contribute very little. Similar studies for other experiments, namely XENON100 32], have been equally successful, confirming that this methodology produces accurate results.

The good agreement achieved for electron recoils also confirms the primordial U/Th contamination of key components, lending more certainty to the calculation of neutron backgrounds from natural radioactivity. The total rate of single elastic scatters in the fiducial volume is 3 events/year in the energy range 5-50 keVnr, which translates to 0.4 events/year in anti-coincidence with the veto for a realistic signal acceptance. This will not limit the sensitivity of the experiment. We also pointed out that neutron time-of-flight can be important to establish scatter multiplicity in large single-phase experiments.

We examined event topologies which challenge the high discrimination power which can be achieved for single-vertex electron recoils. Cherenkov light produced in photomultiplier windows by internal $\beta^{-}$activity or by Compton electrons from background $\gamma$-rays can be detected in coincidence with $\gamma$-ray fiducial scatters, thereby making scintillation pulses appear larger as well as faster - i.e. more similar to nuclear recoils. Dynode glow is another cause of signal-coincident light, although it should affect both S1 and S2 pulses. In this instance photon emission is delayed with respect to the original optical stimulus depending on the input optics configuration and its voltage bias. These mechanisms were identified several decades ago (see, e.g., 33] and references therein). In the context of dark matter searches, tests conducted at Imperial College in the early 1990s confirmed that dynode glow and Cherenkov light were observed when two photomultipliers face each other without an intervening scintillator 34]; subsequently, the NAIAD experiment at Boulby documented a population of fast noise events $(\tau<100 \mathrm{~ns})$ which were attributed to dynode glow (see Fig. 1 in Ref. 35]). More recently, a WIMP search with $\mathrm{CaF}_{2}(\mathrm{Eu})$ scintillator at Kamioka Observatory found that the sensitivity below 10 keVee was limited by Cherenkov photons produced in light guides by Compton electrons caused by background $\gamma$-rays 36 . This type of event may be difficult to identify if the light pattern is not too peaked in a single channel. We note that these effects should also concern single-phase experiments such as XMASS 37, MiniCLEAN 38] and DEAP-3600 39].

We discussed also $\gamma$-ray MSSI events and how these degrade the discrimination power of twophase detectors. They pose a real challenge to current experiments and their mitigation should attract significant design effort in future systems. Whereas single-vertex electron recoils have a steep distribution in the S2/S1 discrimination parameter, and their leaking into the nuclear recoil re- 
gion is progressive, this is not the case for MSSI events. Fortunately, both the frequency and severity of these events decreases for lower energies, where more signal is expected.

Under the assumption that the discrimination power eventually observed is not hindered significantly by poor photomultiplier performance or incomplete rejection of MSSI events, ZEPLIN-III should achieve a sensitivity of $\sim 1 \times 10^{-8} \mathrm{pb} \cdot$ year to the scalar WIMP-nucleon elastic cross-section.

\section{Acknowledgements}

The UK groups acknowledge the support of the Science \& Technology Facilities Council (STFC) for the ZEPLIN-III project and for maintenance and operation of the Boulby underground laboratory. LIP-Coimbra acknowledges financial support from Fundação para a Ciência e a Tecnologia (FCT) through project grant CERN/FP/116374/2010 and postdoctoral grants SFRH/BPD/27054/2006, SFRH/BPD/47320/2008 and SFRH/BPD/63096/2009. The ITEP group acknowledges support from the Russian Foundation of Basic Research (grant 08-02-91851 KO_a) and Rosatom (contract H.4e.45.90.10.1053 from 03-02-2010). We are also grateful for support provided jointly to ITEP and Imperial from the UK Royal Society. ZEPLIN-III is hosted by Cleveland Potash Ltd (CPL) at the Boulby Mine and we thank CPL management and staff for their long-standing support. We also express our gratitude to the Boulby facility staff for their dedication. The University of Edinburgh is a charitable body registered in Scotland (SC005336).

\section{References}

[1] B. A. Dolgoshein, V. N. Lebedenko, B. U. Rodionov, JETP Lett. 11 (1970) 351.

[2] H. M. Araújo, et al., Astropart. Phys. 26 (2006) 140.

[3] D. Y. Akimov, et al., Astropart. Phys. 27 (2007) 46.

[4] D. Y. Akimov, et al., Astropart. Phys. 34 (2010) 151.

[5] C. Ghag, et al., Astropart. Phys. 35 (2011) 76.

[6] V. N. Lebedenko, et al., Phys. Rev. D 80 (2009) 052010.

[7] V. N. Lebedenko, et al., Phys. Rev. Lett. 103 (2009) 151302 .

[8] D. Y. Akimov, et al., Phys. Lett. B 692 (2010) 180.

[9] B. Edwards, et al., Astropart. Phys. 30 (2008) 54.

[10] G. J. Alner, et al., Astropart. Phys. 28 (2007) 287.

[11] J. Angle, et al., Phys. Rev. Lett. 100 (2008) 021303.

[12] E. Aprile, et al., Phys. Rev. Lett. 105 (2010) 131302

[13] D. N. McKinsey, et al., J. Phys.: Conf. Ser. 203 (2010) 012026 .

[14] M. Horn, et al. arXiv:1106.0694.
[15] D. Lewin, et al., http://hepwww.rl.ac.uk/ukdmc/Radioactivity/.

[16] S. Agostinelli, et al., Nucl. Instrum. Meth. A 506 (2003) 250.

[17] B. Edwards, Ph.D. thesis, Imperial College London (2009).

[18] D.-M. Mei, Z.-B. Yin, S. R. Elliot, Astropart. Phys. 31 (2009) 417.

[19] P. Collon, et al., Nucl. Instrum. Meth. B 123 (1997) 122.

[20] J. Gavrilyuk, et al., in: Proc. First Int. Workshop on Nonaccelerator New Physics (NANP 97), 1997, Phys. Atom. Nucl. 61:1287-1292 (1998); Yad. Fiz. 61: 13891394 (1998).

[21] H. Sievers, NDS 62,271, National Nuclear Data Center, NuDat 2 database (www.nndc.bnl.gov/nudat2).

[22] M. Carson, et al., Nucl. Instrum. Meth. A 548 (2005) 418.

[23] H. M. Araújo, et al., Astropart. Phys. 29 (2008) 471.

[24] A. Lindote, et al., Astropart. Phys. 31 (2009) 366.

[25] W. B. Wilson, et al., SOURCES 4A: A code for calculating $(\alpha, \mathrm{n})$, spontaneous fission, and delayed neutron sources and spectra, Tech. Rep. LA-13693, LANL (1999).

[26] W. B. Wilson, et al., SOURCES 4C: A code for calculating $(\alpha, \mathrm{n})$, spontaneous fission, and delayed neutron sources and spectra, Tech. Rep. LA-UR-02-1839, LANL (2002).

[27] M. Carson, et al., Astropart. Phys. 21 (2004) 667.

[28] M. Herman, et al., NDS 108 (2007) 2655.

[29] V. Tomasello, V. A. Kudryavtsev, M. Robinson, Nucl. Instrum. Meth. A 595 (2008) 431.

[30] P. F. Smith, et al., Astropart. Phys. 22 (2005) 409.

[31] E. Tziaferi, et al., Astropart. Phys. 27 (2007) 326.

[32] E. Aprile, et al.ArXiv:1101.3866v1.

[33] A. G. Wright, J. Phys. E: Sci. Instrum. 16 (1983) 300.

[34] J. Li, Tech. rep., Imperial College London (1991).

[35] G. J. Alner, et al., Phys. Lett. B 616 (2005) 17.

[36] C. E. Shimizu, et al., Phys. Lett. B 633 (2006) 195.

[37] Y. Suzuki, arXiv:hep-ph/0008296v1.

[38] D. N. McKinsey, et al., Nuc. Phys. B (Proc. Suppl.) 173 (2007) 152 .

[39] M. Boulay, B. Cai, J. Phys.: Conf. Ser. 136 (2008) 042081 . 